\title{
HWANG'S QUASI-POWER-THEOREM IN DIMENSION TWO
}

\author{
CLEMENS HEUBERGER
}

\begin{abstract}
In a frequently used theorem, H.-K. Hwang proved convergence rates for the central limit theorem of a class of random variables whose moment generating function has a "quasipower" structure. We generalise this result to random vectors of two variables.
\end{abstract}

Gaussian laws in large random combinatorial structures are a frequently observed pattern. In his "quasi-power-theorem", Hwang [2] proved asymptotic normality for a certain class of random variables whose moment generating function satisfies an asymptotic expression which is almost (apart from an error term) of the form $e^{W_{n}(s)}$. He also includes the convergence rates, cf. Theorem 1 below. His result turned out to be particularly useful and frequently used.

The purpose of this note is to provide a version of Hwang's theorem in the case of random vectors of dimension 2, again including the convergence rate. For this, we use a two-dimensional Berry-Esseen-estimate proved by Sadikova [3], cf. Lemma 3.

Although there is a generalisation of Sadikova's result to higher dimensions by Gamkrelidze [1], it seems to be non-trivial to use it for a further generalisation of the quasi-power theorem to higher dimensions.

We will use boldface letters for vectors and $\|\cdot\|$ will denote the maximum norm $\|\mathbf{s}\|=\max \left\{\left|s_{j}\right|\right\}$. Hwang's result is the following.

Theorem 1 (Hwang [2]). Let $\left\{\Omega_{n}\right\}_{n \geq 1}$ be a sequence of integral random variables. Suppose that the moment generating function satisfies the asymptotic expression

$$
M_{n}(s):=\mathbb{E}\left(e^{\Omega_{n} s}\right)=\sum_{m \geq 0} \mathbb{P}\left(\Omega_{n}=m\right) e^{m s}=e^{W_{n}(s)}\left(1+O\left(\kappa_{n}^{-1}\right)\right),
$$

the O-term being uniform for $|s| \leq \tau, s \in \mathbb{C}, \tau>0$, where

(1) $W_{n}(s)=u(s) \phi(n)+v(s)$, with $u(s)$ and $v(s)$ analytic for $|s| \leq \tau$ and independent of $n$; and $u^{\prime \prime}(0) \neq 0$;

(2) $\lim _{n \rightarrow \infty} \phi(n)=\infty$;

(3) $\lim _{n \rightarrow \infty} \kappa_{n}=\infty$.

Then the distribution of $\Omega_{n}$ is asymptotically normal, i.e.,

$$
\mathbb{P}\left(\frac{\Omega_{n}-u^{\prime}(0) \phi(n)}{\sqrt{u^{\prime \prime}(0) \phi(n)}}<x\right)=\Phi(x)+O\left(\frac{1}{\sqrt{\phi(n)}}+\frac{1}{\kappa_{n}}\right)
$$

uniformly with respect to $x, x \in \mathbb{R}$, where $\Phi$ denotes the standard normal distribution

$$
\Phi(x)=\frac{1}{\sqrt{2 \pi}} \int_{-\infty}^{x} \exp \left(-\frac{1}{2} y^{2}\right) d y .
$$

We intend to prove the following 2-dimensional version of Theorem 1.

Date: July 30, 2007.

2000 Mathematics Subject Classification. 60F05; 60C05.

This paper was partly written while C. Heuberger was a visitor at the Center of Experimental Mathematics at the University of Stellenbosch. He thanks the center for its hospitality. He is also supported by the Austrian Science Foundation FWF, project S9606, that is part of the Austrian National Research Network "Analytic Combinatorics and Probabilistic Number Theory." 
Theorem 2. Let $\left\{\boldsymbol{\Omega}_{n}\right\}_{n \geq 1}$ be a sequence of two dimensional integral random vectors. Suppose that the moment generating function satisfies the asymptotic expression

$$
M_{n}(\mathbf{s}):=\mathbb{E}\left(e^{\left\langle\boldsymbol{\Omega}_{n}, \mathbf{s}\right\rangle}\right)=\sum_{\mathbf{m} \geq 0} \mathbb{P}\left(\boldsymbol{\Omega}_{n}=\mathbf{m}\right) e^{\langle\mathbf{m}, \mathbf{s}\rangle}=e^{W_{n}(\mathbf{s})}\left(1+O\left(\kappa_{n}^{-1}\right)\right),
$$

the O-term being uniform for $\|\mathbf{s}\|_{\infty} \leq \tau, \mathbf{s} \in \mathbb{C}^{2}, \tau>0$, where

(1) $W_{n}(\mathbf{s})=u(\mathbf{s}) \phi(n)+v(\mathbf{s})$, with $u(\mathbf{s})$ and $v(\mathbf{s})$ analytic for $\|\mathbf{s}\| \leq \tau$ and independent of $n$; and the Hessian $H_{u}(\mathbf{0})$ of $u$ at the origin is nonsingular;

(2) $\lim _{n \rightarrow \infty} \phi(n)=\infty$;

(3) $\lim _{n \rightarrow \infty} \kappa_{n}=\infty$.

Then, the distribution of $\boldsymbol{\Omega}_{n}$ is asymptotically normal, i.e.,

$$
\mathbb{P}\left(\frac{\boldsymbol{\Omega}_{n}-\operatorname{grad} u(\mathbf{0}) \phi(n)}{\sqrt{\phi(n)}} \leq \mathbf{x}\right)=\Phi_{H_{u}(\mathbf{0})}(\mathbf{x})+O\left(\frac{1}{\sqrt{\phi(n)}}+\frac{1}{\kappa_{n}}\right)
$$

where $\Phi_{\Sigma}$ denotes the distribution function of the two dimensional normal distribution with mean $\mathbf{0}$ and variance-covariance matrix $\Sigma$, i.e.,

$$
\Phi_{\Sigma}(\mathbf{x})=\frac{1}{2 \pi \sqrt{\operatorname{det} \Sigma}} \iint_{\mathbf{y} \leq \mathbf{x}} \exp \left(-\frac{1}{2} \mathbf{y}^{t} \Sigma^{-1} \mathbf{y}\right) d \mathbf{y}
$$

where $\mathbf{y} \leq \mathbf{x}$ means $y_{1} \leq x_{1}$ and $y_{2} \leq x_{2}$.

The proof of Theorem 2 relies on the following two-dimensional Berry-Esseen-inequality.

Lemma 3 (Sadikova [3]). Let $\mathbf{X}$ and $\mathbf{Y}$ be two-dimensional random vectors with distribution functions $F$ and $G$ and characteristic functions $f$ and $g$, respectively.

Let

$$
\hat{f}\left(s_{1}, s_{2}\right)=f\left(s_{1}, s_{2}\right)-f\left(s_{1}, 0\right) f\left(0, s_{2}\right), \quad \hat{g}\left(s_{1}, s_{2}\right)=g\left(s_{1}, s_{2}\right)-g\left(s_{1}, 0\right) g\left(0, s_{2}\right),
$$

and

$$
A_{1}=\sup _{x_{1}, x_{2}} \frac{\partial G\left(x_{1}, x_{2}\right)}{\partial x_{1}}, \quad A_{2}=\sup _{x_{1}, x_{2}} \frac{\partial G\left(x_{1}, x_{2}\right)}{\partial x_{2}} .
$$

Then for any $T>0$, we have

$$
\begin{aligned}
(2) \sup _{x, y} & |F(x, y)-G(x, y)| \leq \frac{2}{(2 \pi)^{2}} \iint_{\|\mathbf{s}\| \leq T}\left|\frac{\hat{f}\left(s_{1}, s_{2}\right)-\hat{g}\left(s_{1}, s_{2}\right)}{s_{1} s_{2}}\right| d \mathbf{s} \\
& +2 \sup _{x}|F(x, \infty)-G(x, \infty)|+2 \sup _{y}|F(\infty, y)-G(\infty, y)|+\frac{2\left(A_{1}+A_{2}\right)}{T}(3 \sqrt{2}+4 \sqrt{3}) .
\end{aligned}
$$

Proof of Theorem 2. We define $E_{n}(\mathbf{s})$ by the relation $M_{n}(\mathbf{s})=e^{W_{n}(\mathbf{s})}\left(1+E_{n}(\mathbf{s})\right)$ and note that by assumption, $E_{n}(\mathbf{s})=O\left(\kappa_{n}^{-1}\right)$ uniformly for $\|\mathbf{s}\| \leq \tau$. We note that this implies $u(\mathbf{0})=v(\mathbf{0})=0$ and therefore $E_{n}(\mathbf{0})=0$.

Let $\boldsymbol{\mu}_{n}=\phi(n) \operatorname{grad} u(\mathbf{0})$ and $\Sigma=H_{u}(\mathbf{0})$. We define the random vector $\boldsymbol{\Omega}_{n}^{*}=\phi(n)^{-1 / 2}\left(\boldsymbol{\Omega}_{n}-\boldsymbol{\mu}_{n}\right)$ with distribution function $F_{n}(\mathbf{x})$ and characteristic function

$$
f_{n}(\mathbf{s})=M_{n}\left(i \phi(n)^{-1 / 2} \mathbf{s}\right) \exp \left(-i \phi(n)^{-1 / 2}\left\langle\boldsymbol{\mu}_{n}, \mathbf{s}\right\rangle\right)=\exp \left(-\frac{1}{2} \mathbf{s}^{t} \Sigma \mathbf{s}+W_{n}^{*}(\mathbf{s})\right)\left(1+E_{n}\left(i \phi(n)^{-1 / 2} \mathbf{s}\right)\right)
$$

with

$$
W_{n}^{*}(\mathbf{s})=u\left(i \phi(n)^{-1 / 2} \mathbf{s}\right) \phi(n)+v\left(i \phi(n)^{-1 / 2} \mathbf{s}\right)-i \phi(n)^{-1 / 2}\left\langle\boldsymbol{\mu}_{n}, \mathbf{s}\right\rangle+\frac{1}{2} \mathbf{s}^{t} \Sigma \mathbf{s} .
$$


We consider the univariate analytic functions $u_{j}\left(s_{j}\right), v_{j}\left(s_{j}\right), E_{n, j}\left(s_{j}\right)$ for $j=1,2$ and the bivariate analytic functions $u_{0}(\mathbf{s}), v_{0}(\mathbf{s}), E_{n, 0}(\mathbf{s})$ satisfying

$$
\begin{aligned}
u(\mathbf{s}) & =\langle\operatorname{grad} u(\mathbf{0}), \mathbf{s}\rangle+\frac{1}{2} \mathbf{s}^{t} \Sigma \mathbf{s}+s_{1}^{2} u_{1}\left(s_{1}\right)+s_{2}^{2} u_{2}\left(s_{2}\right)+s_{1} s_{2} u_{0}(\mathbf{s}), \\
v(\mathbf{s}) & =v_{1}\left(s_{1}\right)+v_{2}\left(s_{2}\right)+s_{1} s_{2} v_{0}(\mathbf{s}), \\
E_{n}(\mathbf{s}) & =E_{n, 1}\left(s_{1}\right)+E_{n, 2}\left(s_{2}\right)+s_{1} s_{2} E_{n, 0}(\mathbf{s}), \\
0 & =u_{j}(0)=v_{j}(0)=E_{n, j}(0), \quad j \in\{1,2\}, \\
0 & =u_{0}(\mathbf{0}) .
\end{aligned}
$$

Let $c$ be a positive constant less than $\max \{\tau / 2,1\}$ which will be specified later and let $T_{n}=$ $c \sqrt{\phi(n)}$. With these notations, we have

$$
\begin{aligned}
W_{n}^{*}(\mathbf{s})= & -s_{1}^{2} u_{1}\left(i \phi(n)^{-1 / 2} s_{1}\right)-s_{2}^{2} u_{2}\left(i \phi(n)^{-1 / 2} s_{2}\right)-s_{1} s_{2} u_{0}\left(i \phi(n)^{-1 / 2} \mathbf{s}\right) \\
& +v_{1}\left(i \phi(n)^{-1 / 2} s_{1}\right)+v_{2}\left(i \phi(n)^{-1 / 2} s_{2}\right)-\frac{s_{1} s_{2}}{\phi(n)} v_{0}\left(i \phi(n)^{-1 / 2} \mathbf{s}\right) \\
= & O\left(\rho_{n}(\mathbf{s})\right) .
\end{aligned}
$$

for $\|\mathbf{s}\|<T_{n}$, where

$$
\rho_{n}(\mathbf{s}):=\frac{\|\mathbf{s}\|^{3}+\|\mathbf{s}\|}{\sqrt{\phi(n)}} .
$$

Since $E_{n}\left(\left(s_{1}, 0\right)\right)=E_{n, 1}\left(s_{1}\right)=O\left(\kappa_{n}^{-1}\right)$ and $E_{n}\left(\left(0, s_{2}\right)\right)=E_{n, 2}\left(s_{2}\right)=O\left(\kappa_{n}^{-1}\right)$, we also have $s_{1} s_{2} E_{n, 0}(\mathbf{s})=O\left(\kappa_{n}^{-1}\right)$. By Cauchy's integral formula, we also get $E_{n, 0}(\mathbf{s})=O\left(\kappa_{n}^{-1}\right)$ for $\|\mathbf{s}\|<\tau / 2$. Similarly, we have $E_{n, j}=s_{j} O\left(\kappa_{n}^{-1}\right)$ for $\|\mathbf{s}\|<\tau / 2$ and $j=1,2$.

Note that

$$
\lim _{n \rightarrow \infty} f_{n}(\mathbf{s})=\exp \left(-\frac{1}{2} \mathbf{s}^{t} \Sigma \mathbf{s}\right)=: g(\mathbf{s})
$$

for $\mathbf{s} \in \mathbb{R}^{2}$, which implies that in distribution, $\boldsymbol{\Omega}_{n}^{*}$ converges to the normal distribution with mean zero and variance-covariance matrix $\Sigma$. Although we have to refine our estimates for applying Lemma 3, we conclude immediately that $\Sigma$ is positive definite (since it is nonsingular).

We now estimate $\hat{f}(\mathbf{s})$ as defined in (1) for $\|\mathbf{s}\|<T_{n}$ :

$$
\begin{aligned}
\hat{f}(\mathbf{s})= & \exp \left(-\frac{1}{2} \mathbf{s}^{t} \Sigma \mathbf{s}\right) \exp \left(W_{n}^{*}(s)\right) \\
& \times\left(1+E_{n, 1}\left(i \phi(n)^{-1 / 2} s_{1}\right)+E_{n, 2}\left(i \phi(n)^{-1 / 2} s_{2}\right)-s_{1} s_{2} \phi(n)^{-1} E_{n, 0}\left(i \phi(n)^{-1 / 2} \mathbf{s}\right)\right. \\
& \quad-\exp \left(s_{1} s_{2}\left(\sigma_{12}+u_{0}\left(i \phi(n)^{-1 / 2} \mathbf{s}\right)+\phi(n)^{-1} v_{0}\left(i \phi(n)^{-1 / 2} \mathbf{s}\right)\right)\right) \\
& \left.\quad \times\left(1+E_{n, 1}\left(i \phi(n)^{-1 / 2} s_{1}\right)+E_{n, 2}\left(i \phi(n)^{-1 / 2} s_{2}\right)+E_{n, 1}\left(i \phi(n)^{-1 / 2} s_{1}\right) E_{n, 2}\left(i \phi(n)^{-1 / 2} s_{2}\right)\right)\right) \\
= & \exp \left(-\frac{1}{2} \mathbf{s}^{t} \Sigma \mathbf{s}\right) \exp \left(W_{n}^{*}(s)\right)\left(1-\exp \left(s_{1} s_{2} \sigma_{12}\right)\left(1+s_{1} s_{2} O\left(\|\mathbf{s}\| \phi(n)^{-1 / 2}\right) \exp \left(O\left(\rho_{n}(\mathbf{s})\right)\right)\right)\right) \\
& \quad \times\left(1+E_{n, 1}\left(i \phi(n)^{-1 / 2} s_{1}\right)+E_{n, 2}\left(i \phi(n)^{-1 / 2} s_{2}\right)\right) \\
& +s_{1} s_{2} \exp \left(-\frac{1}{2} \mathbf{s}^{t} \Sigma \mathbf{s}+O\left(\rho_{n}(\mathbf{s})\right)\right) O\left(\kappa_{n}^{-1} \phi(n)^{-1}\right) \\
& +s_{1} s_{2} \exp \left(-\frac{1}{2}\left(\sigma_{11} s_{1}^{2}+\sigma_{22} s_{2}^{2}\right)+O\left(\rho_{n}(\mathbf{s})\right)\right) O\left(\kappa_{n}^{-2} \phi(n)^{-1}\right) \\
= & \exp \left(-\frac{1}{2} \mathbf{s}^{t} \Sigma \mathbf{s}\right)\left(1-\exp \left(s_{1} s_{2} \sigma_{12}\right)\right) \\
& +s_{1} s_{2} \exp \left(-\frac{1}{2} \mathbf{s}^{t} \Sigma \mathbf{s}+O\left(\rho_{n}(\mathbf{s})\right)\right) O\left(\kappa_{n}^{-1} \phi(n)^{-1}\right) \\
& +s_{1} s_{2} \exp \left(-\frac{1}{2}\left(\sigma_{11} s_{1}^{2}+\sigma_{22} s_{2}^{2}\right)+O\left(\rho_{n}(\mathbf{s})\right)\right) O\left(\kappa_{n}^{-1}+\rho_{n}(\mathbf{s})\right)
\end{aligned}
$$

where the inequality $|\exp (w)-1| \leq|w| \exp (|w|)$ for all complex $w$ has been used repeatedly. 
In order to apply Lemma 3 , we estimate $|\hat{f}(\mathbf{s})-\hat{g}(\mathbf{s})| /\left|s_{1} s_{2}\right|$ for $\|\mathbf{s}\|<T_{n}$ :

$$
\begin{aligned}
\left|\frac{\hat{f}(\mathbf{s})-\hat{g}(\mathbf{s})}{s_{1} s_{2}}\right|=\exp \left(-\frac{1}{2} \mathbf{s}^{t} \Sigma \mathbf{s}+O\left(\rho_{n}(\mathbf{s})\right)\right) O\left(\kappa_{n}^{-1} \phi(n)^{-1}\right) & \\
& +\exp \left(-\frac{1}{2}\left(\sigma_{11} s_{1}^{2}+\sigma_{22} s_{2}^{2}\right)+O\left(\rho_{n}(\mathbf{s})\right)\right) O\left(\kappa_{n}^{-1}+\rho_{n}(\mathbf{s})\right) .
\end{aligned}
$$

We choose $c$ sufficiently small such that for $\|\mathbf{s}\|<T_{n}$ :

$$
\left|\frac{\hat{f}(\mathbf{s})-\hat{g}(\mathbf{s})}{s_{1} s_{2}}\right|=\exp \left(-\frac{1}{4} \mathbf{s}^{t} \Sigma \mathbf{s}\right) O\left(\kappa_{n}^{-1} \phi(n)^{-1}\right)+\exp \left(-\frac{1}{4}\left(\sigma_{11} s_{1}^{2}+\sigma_{22} s_{2}^{2}\right)\right) O\left(\kappa_{n}^{-1}+\rho_{n}(\mathbf{s})\right) .
$$

For a constant $k \geq 0$, we have

$$
\int_{0}^{\infty} \exp \left(-x^{2}\right) x^{k} d x=\frac{1}{2} \Gamma\left(\frac{k+1}{2}\right)
$$

and we conclude that

$$
\iint_{\|\mathbf{s}\| \leq T_{n}}\left|\frac{\hat{f}(\mathbf{s})-\hat{g}(\mathbf{s})}{s_{1} s_{2}}\right| d \mathbf{s}=O\left(\frac{1}{\sqrt{\phi(n)}}+\frac{1}{\kappa_{n}}\right) .
$$

For estimating the second and the third summand in (2) we simply use Hwang's result in dimension 1 (Theorem 1) to see that they are also bounded by $O\left(\phi(n)^{-1 / 2}+\kappa_{n}^{-1}\right)$. The fourth summand is bounded by $O\left(\phi(n)^{-1 / 2}\right)$.

\section{REFERENCES}

1. N. G. Gamkrelidze, A multidimensional generalization of Esseen's inequality for distribution functions, Theory Probab. Appl. 22 (1977), 877-880.

2. H.-K. Hwang, On convergence rates in the central limit theorems for combinatorial structures, European J. Combin. 19 (1998), 329-343. MR 99c:60014

3. S. M. Sadikova, On two-dimensional analogues of an inequality of Esseen and their application to the central limit theorem, Theory Probab. Appl. XI (1966), 325-335, English Translation of the paper in Teor. Verojatnost. i Primenen.

Institut für Mathematik B, Technische Universität Graz, Austria

E-mail address: clemens.heuberger@tugraz.at 